Review

\title{
The Emerging Roles of Microparticles in Diabetic Nephropathy
}

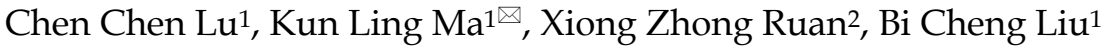 \\ 1. Institute of Nephrology, Zhong Da Hospital, School of Medicine, Southeast University, Nanjing City, Jiangsu Province, China; \\ 2. Centre for Nephrology, University College London (UCL) Medical School, Royal Free Campus, UK. \\ $\triangle$ Corresponding author: Kun Ling Ma, Institute of Nephrology, Zhong Da Hospital, Southeast University School of Medicine, NO.87, Ding Jia Qiao Road, \\ Nanjing City, Jiangsu Province, China, 210009. Tel: 008625 83262442, E-mail: klma05@163.com \\ () Ivyspring International Publisher. This is an open access article distributed under the terms of the Creative Commons Attribution (CC BY-NC) license \\ (https://creativecommons.org/licenses/by-nc/4.0/). See http://ivyspring.com/terms for full terms and conditions.
}

Received: 2017.05.22; Accepted: 2017.07.23; Published: 2017.09.05

\begin{abstract}
Microparticles (MPs) are a type of extracellular vesicles (EVs) shed from the outward budding of plasma membranes during cell apoptosis and/or activation. These microsized particles then release specific contents (e.g., lipids, proteins, microRNAs) which are active participants in a wide range of both physiological and pathological processes at the molecular level, e.g., coagulation and angiogenesis, inflammation, immune responses. Research limitations, such as confusing nomenclature and overlapping classification, have impeded our comprehension of these tiny molecules. Diabetic nephropathy (DN) is currently the greatest contributor to end-stage renal diseases (ESRD) worldwide, and its public health impact will continue to grow due to the persistent increase in the prevalence of diabetes mellitus (DM). MPs have recently been considered as potentially involved in DN onset and progression, and this review juxtaposes some of the research updates about the possible mechanisms from several relevant aspects and insights into the therapeutic perspectives of MPs in clinical management and pharmacological treatment of DN patients.
\end{abstract}

Key words: microparticles; diabetic nephropathy; transcellular crosstalk; insulin resistence; endoplasmic reticulum stress; vascular endothelial growth factor $\mathrm{A}$.

\section{Introduction of MPs}

Microparticles (MPs) were first described as "platelet dust" in the early 1960s s $^{[1]}$, and were assumed to be biologically nonsignificant shortly after their discovery. MPs are one type of extracellular vesicles (EVs), which has been a research hotpot in recent years and includes two other types: "exosomes" and "apoptotic bodies". Established science has investigated multiple effects of EVs, but most work in this field has focused on exosomes. In published studies, MPs and exosomes are often confused and termed as EVs, though they are actually entities with several disparate characteristics[2]. Moreover, some documents have been reported contradictory characters and/or functions of MPs derived from the same cell type. Such phenomena, to some extent, based on an inexplicit classification, are likely to be a consequence of our incomplete understanding of EVs and lack of standard isolating protocols. In view of this, Gould, S.J. et al. ${ }^{[3]}$ have offered some suggestions for this nomenclature complexity: 1) State the explicit use of the vesicles separately, choose the terms on the basis of certificated statement, and make sure these chosen terms are consistently applied throughout the whole text; 2) Introduce the relationship between these vesicles and their corresponding collecting $\operatorname{method}(\mathrm{s})$, also for obtaining and/or storing biological fluids containing these vesicles before isolation; 3) Use the term "extracellular vesicle" (EV) as a generic term as far as possible and as a keyword in all announcement and publications.

MPs, sometimes termed as "microvesicles" (MVs), are 0.1- to 1.0- $\mu \mathrm{m}$ spherical-like structures, which are encapsulated by a lipid bilayer that typically possess membrane and cytoplasmic contents 
(including lipids, proteins, mRNA and/or miRNA, and even DNA) of their parent cells and are reported to take up materials in the microenvironment where they are generated ${ }^{[4]}$. These bioactive substances enable MPs to exert their effects on fundamental biological processes in a pleiotropic manner. Under physiologic circumstances, MPs in the peripheral blood are derived mostly from platelets and endothelial cells (ECs), whereas in pathological contexts, numerous kinds of cells have been observed to release MPs, including erythrocytes, monocytes, lymphocytes and many other cell types. A multitude of studies have focused on plasma MPs due to their easier accessibility, whereas MPs from human body fluids (e.g. urine) have also been considered to potentially indicate the status of intrinsic cells, tissues and/or organs. The underlying mechanism that reportedly regulates the formation and release of MPs remains to be fully elucidated, but there has been some consensus: the entire MP-releasing process can be initiated by different forms of stimuli (e.g., inflammation) and mediated by a series of mechanical and/or biochemical factors, leading to calpain activation and phosphatase inhibition. Then, via enzymes called flippases, floppases and scramblases, through cytoskeletal reorganization and translocation of phosphatidylserine (PS) from the inner monolayer of the plasma membrane to the surface of MPs[5], and results in the final vesiculation and outward budding of the plasma membrane. In addition, Rho-associated kinase (ROCK) has been proven to be relevant to the shedding of apoptotic MPs[ ${ }^{[6]}$ (see Figure 1).

Once released from parent cells, MPs must dock on the surface of target cells to exert specific functions, where they can initiate signalling events ${ }^{[7]}$ or be further internalized by recipients via membrane fusion or endocytosis. Having been implicated in both beneficial (e.g., prevents apoptosis ${ }^{[8]}$ ) and detrimental reactions (e.g., inflammation ${ }^{[9]}$ ), the uptake of MPs may be facilitated not only by their cargo but also by the characteristics of target cells and result in phenotypic and/or functional changes in the latter. Otherwise, MPs are likely to be eliminated by phagocytic cells, which are vital to the daily clearance of apoptotic cells and metabolic wastes and are closely associated with inflammatory processes. Recently, macrophages have been found to release both microvesicles and insulin-like growth factor-1 (IGF-1) during phagocytosis or responses to inflammatory cytokines; IGF-1 seems capable of enhancing the uptake of MVs by ECs, which critically decreases the magnitude of epithelial inflammation in vivo[10]. In addition, the clearance rate of MPs varies among different cellular origins, surface receptors and/or adhesion molecules because they bind to these molecules and external stress promotes their release ${ }^{[11,}$ 12].

A variety of isolation techniques has been used for the qualitative and/or quantitative detection of MPs. The most common starting step is centrifugation to obtain cell-free media or platelet-free plasma samples, after which specific fluorescent-conjugated antibodies to cell-surface antigens or Annexin V, which labels externalized phosphatidylserine, are used with flow cytometry for the determination and enumeration of MPs ${ }^{[13]}$.

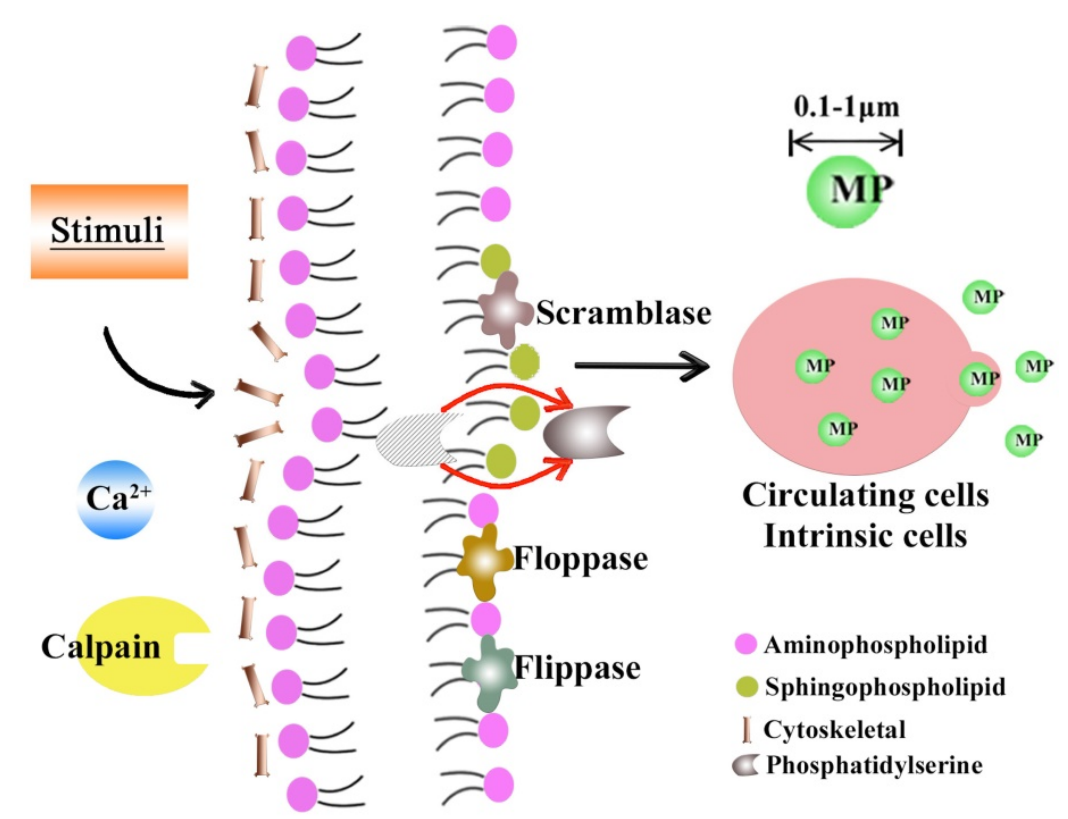

Figure 1. The formation of MPs. When stimulated by external factors (e.g., inflammation, apoptosis), intracellular calpain and phosphatase are, respectively, activated and inhibited, reorganizing the cytoskeleton via three enzymes (flippases, floppases and scramblase) and phosphatidylserine (PS) exposure from the inner monolayer to the MP surface, all of which led to the final vesiculation and outward budding of the plasma membrane. 


\section{MPs as potential biomarkers in DN}

Diabetes mellitus (DM) refers to a heterogeneous group of metabolic disorders that generally manifests as hyperglycaemia. DM is mostly classified into two categories based on their pathogenisis: type $1 \mathrm{DM}$ (T1DM) is resulted from complete deficiency of insulin secretion, while T2DM is characterized by various degrees of insulin resistance (IR) ${ }^{[14]}$. T1DM commonly develops before the age of 30, but an thorough autoimmune destruction of beta cells can develop at any age; T2DM is more typically seen among the elders, though it has an increasing incidence trend in the population of children and young adults[15]. As reviewed[16], there are discrepancies of cellular circulating microparticles (cMPs) patterns between patients with different types and stages (including diabetic complications) of DM: higher levels of PMPs, EMPs, and total annexin V positive microparticles have been shown in T1DM patients; while in T2DM patients, numbers of total MPs and MPs from leukocyte, monocyte, and endothelial cells have increased compared with those of matched controls. In addition, it has been found that elevated circulating levels of various MPs could predict cardio- and vascular outcome of DM patients $[17,18]$.

$\mathrm{DM}$ has reached epidemic proportions worldwide and predisposes patients to develop multiple-organ complications, among which diabetic nephropathy (DN) has taken a vast toll on undesirable renal outcomes ${ }^{19]}$. The major pathological alterations of DN initiate from the excessive deposition of extracellular matrix (ECM), progress to mesangial proliferation and expansion along with growing thickness of the glomerular basement membrane (GBM) and finally develop into renal fibrosis with a complete functional loss. Currently, we monitor the progression of DKD mainly via (micro)albuminuria, GFR levels and/or creatinine clearance to confirm disease status, but in fact, renal injuries are likely to start when patient shows no abnormal clinical indicators ${ }^{[2]}$. Therefore, it is critical to investigate noninvasive and sensitive biomarkers that can predict disease progression.

It has been reported by $\mathrm{Li}, \mathrm{S}$. et al. ${ }^{[21]}$ that circulating total MPs (TMPs) and MPs from certain cell types, including platelets and monocytes, have been observed greatly elevated in patients with T2DM; besides, instead of activated endothelium-derived MPs (EMPs), apoptotic EMPs were significantly increased in these patients, both suggested that changing MPs levels are possibly relevant to DM conditions. There has also been consensus on the fact that CKD individuals have platelet activation and endothelial dysfunction, and as novel markers, PMPs and EMPs can be used to detect these pathological changes and to imply the development of $\mathrm{CKD}^{[22]}$. In the context of $\mathrm{DN}, \mathrm{MP}$ accumulation can be observed in specific organs and has a close correlation with disease severity, but most MPs are detected in the circulating system, while actually the levels of MPs in body fluids also have implicating values. For instance, Lytvyn, Y. et al.[23] have discovered that in T1DM patients, there existed a higher level of urinary podocyte MPs, which suggestes glomerular injury of diabetic incipient stages even earlier than routine biomarkers (albuminuria, nephrin). Coincidentally, Burger et al.[24] found that in reaction to high glucose conditions, podocytes could accelerate MP production and release them into urine, which might be an early indicator of glomerular lesions in DN. With markers such as podocalyxin and nephrin, urinary podocyte-derived MPs can be detected using flow cytometry ${ }^{[25]}$ and indicate kidney injury by displaying elevated levels. In addition to implicating glomerular status, MPs has also been reported to reflect early tubular impairment in T2DM. Dipeptidyl peptidase-IV (DPP-IV) is a membrane protein that is secreted from tubular epithelial cells and is bound by microparticles in urine. Thus, increasing excretion of MP-bound DPP-IV is associated with renal damage in $\mathrm{DKD}{ }^{[26]}$. In short, there is a great possibility that urinary MPs be a new indicator for $\mathrm{DN}$ because of their noninvasive availability and potential to assess the risk of other complications.

\section{MPs in DN pathogenesis}

Hyperglycaemia is generally thought to be one of the most important attributes of developing diabetic complications because it causes injuries to cells and organs in various ways, e.g., inducing cell apoptosis[27]. Controlling blood glucose levels has been proven to slow the progression of DN and loss of kidney function[28]. However, some patients with uncontrolled DM are not inclined to develop diabetic renal complications, which suggest that chronic high glucose is necessary but not sufficient for the progression of DN. There are still several other factors that have proposed to be implicated in the pathogenesis of $\mathrm{DN}$, such as lipid disorders, endothelial dysfunction, systemic inflammation. Despite the general involvement of MPs in promoting inflammation, recently Eirin et al. ${ }^{[29]}$ have found that mesenchymal stem cell (MSC)-derived EVs can exert renoprotective effects by activating anti-inflammatory signalling; overall, however, with demonstrable increases in these abnormal contexts, MPs are supposed to be potentially active players in DN via 
the mechanisms proposed below.

\section{MPs in transcellular crosstalk}

As in the macro world, where media can be used for the exchange of information, cells can communicate with each other via different substances and signals. There is mounting evidence suggesting that MPs are potent conveyers of information between cells and can accelerate or retard biological reactions. After exiting original cells or tissues, MPs can be recognized by target cells when arriving at pre-set sites of action. Under lipotoxic stress, adipocytes have been observed to release a "find $\mathrm{me}^{\text {" signal, i.e., }}$ Annexin V-positive MPs, which acts as central chemoattractants and causes a state of chronic low-grade inflammation [30]. Released MPs can alter the intrinsic features of their recipient cells. A recent study indicated that MPs from monocytes and endothelial cells can induce an inflammatory phenotype in podocytes, which leads to glomerular inflammation in vivo and promotes the development of proteinuriaa ${ }^{[31]}$.

As reviewed[4], MPs mediate transcellular crosstalk mainly through two possible mechanisms: 1) Activation of target cell receptors with bioactive molecules on the surface (namely, receptor-ligand interaction), which leads to subsequent cascade responses; and 2) Delivery of bioactive protein, lipid and genetic content (mRNA, microRNA or even DNA) after internalized by target cells. Take MicroRNAs (hereinafter referred to miRNAs) as an example. It has long been reported that miRNAs are a significant cargo contained by MPs and are related to the diabetic disruption of internal homeostasis, e.g., after controlling treatment of high blood glucose levels, endothelial cells produce MPs with decreased amounts of miR-126, which can induce endothelial repair ${ }^{[32]}$. Certain miRNAs, such as miRNA-29a, have even been able to protect against podocyte injury and restore renal homeostasis in $\mathrm{DN}[33]$, although whether this effect is MP-mediated has not yet been determined. EVs released by human islets can be internalized into islet endothelial cells, resulting in insulin mRNA transfer, apoptotic resistance, and angiogenesis in vitro. These activities may indicate the molecular mechanisms of cross-talk between beta cells and endothelium ${ }^{[34]}$. In addition, endothelial MPs from cultured ECs have been found to contain NADPH oxidase subunits, which enables these ECs to produce ROS and induce downstream effects on EC signalling without direct physical contact[35]. This observation may be of particular relevance in the in vivo setting where physical interactions between MPs and the endothelium may be limited, such as the interaction between podocytes and glomerular endothelial cells (GECs) which is restricted by glycocalyx covering.

\section{MPs and insulin resistance}

Defined as cells with impaired capacities to respond to the glucose-lowering effects of insulin, insulin resistance (IR) is generally thought to be an important feature of T2DM, whereas increased IR can also be detected in patients with T1DM and microalbuminuria; furthermore, the severity of IR is largely associated with DN progression [36]. With insulin receptors mostly expressed on tubular cells and podocytes in the kidney, DM-related hyperglycaemia, inflammation and dyslipidaemia all can contribute to IR in these two parts of the kidney and result in subsequent injury and/or dysfunction ${ }^{[37]}$.

EVs from adipose tissues (ATs), referred to as AT-EVs, has been reported to intervene in insulin signalling and contribute to local IR in organs such as liver[38] and skeletal muscle ${ }^{[39]}$, which may depend on the transfer of their adipokine content ${ }^{[40]}$. Additionally, a potential role for AT-EVs has been proposed in the crosstalk between adipocytes and macrophages, which would further aggravate systemic IR ${ }^{[41]}$. Akt (known as protein kinase B) is the major downstream effector of PI3K (phosphatidylinositol 3-kinase), and insulin signalling is mostly mediated via the PI3K/Akt pathway. Given the adipose tissue macrophages (ATM) M1 and M2 phenotypes, MPs derived from M1 macrophages has been found to reduce Akt phosphorylation stimulated by insulin through enhancing NF-KB activation; in addition, MPs block glucose uptake by hampering glucose transporter (GLUT4) translocation to the cell surface ${ }^{[42]}$. However, no reports have yet described IR caused by MPs in DN, and even reverse outcomes have been described in that the EMP levels are not elevated to IR conditions ${ }^{[43]}$.

\section{MPs and organelle oxidative stress}

Mitochondria and endoplasmic reticulum (ER) are both essential contributors to maintaining intracellular homeostasis, and mitochondrial dysfunction and ER stress have recently emerged as a potential mechanisms involved in progressive $\mathrm{DN}$ deterioration.

Physiologically, the oxidative respiratory chain (also referred to as the electron transport chain, ETC) embedded in the inner membrane of the mitochondria is the main source of cellular reactive oxygen species (ROS), which are innoxious when kept at normal levels. However, pathologic factors, e.g., chronic exposure to high glucose levels (as occurs in diabetes) and disrupted mitochondria, especially in the 
glomerular endothelium[44], may result in overload of ROS products and oxidative stress, which have been recognized as one of the major culprits to DN kidney injuries. ER is a significant site for $\mathrm{Ca}^{2+}$ deposition, lipid synthesis and protein folding. Accumulating misfolded and unfolded proteins in the ER lumen, along with $\mathrm{Ca}^{2+}$ disorder, can induce ER stress and activate the unfolded protein response (UPR). Typically, the UPR is a protective mechanism during its early phases, but in contrast to the original intention of handling protein overload, prolonged UPR activation results from persistent hyperglycaemia and can promote podocyte apoptosis under diabetic conditions ${ }^{[45]}$. However, it has been discovered that hepatocytes confronted with lipotoxicity would release MPs containing mtDNA and oxidized DNA like intact mitochondria, which promotes proinflammatory cytokine secretion, e.g., TNF- $\alpha$ and IL-1 $\beta$, that can further enhance hepatic damage[46].

Mitochondria and the ER are not independent intracellular organelles, instead they exchange metabolites and signalling molecules at the 'mitochondria-associated ER membrane (MAM)' interfaces. Although some studies have suggested that mitochondrial ROS production is downstream of ER stress ${ }^{[47]}$, it has also been proposed that an upset of ER homoeostasis is subsequent to mitochondrial dysfunction in certain metabolic diseases. Due to the close interactions between these organelles, the exact cause-and-effect relationship between mitochondrial dysfunction and ER stress has not yet been determined. Zainab Safiedeen et al. conducted detailed analyses of the crosstalk between ER and mitochondria in the regulation of oxidative stress ${ }^{[47]}$ and found that MPs from apoptotic T cells can act on Fas and LDL-R and induce cytosolic ROS production via the neutral sphingomyelinase (SMase) pathway. Increased cytosolic ROS activates ER stress through the interactions between ER and mitochondria. In addition, neutral SMase activation can directly induce ER stress, which in turn increases both cytosolic and mitochondrial ROS. All of these lead to the decreased bioavailability of NO and subsequent impairment of endothelium-dependent vasorelaxation.

\section{MPs and vascular endothelial growth factor}

The vascular endothelial growth factor (VEGF) family consists of at least seven variants with separated properties concerning angiogenesis - for instance, VEGF-B neutralization has potentially therapeutic values in T2DM by ameliorating IR and enhancing glucose tolerance ${ }^{[48]}-$ whereas the term "VEGF" typically refers to its "VEGF-A" isoform.

Despite the possible extra origins from distal/collecting duct tubular cells and activated mesangial cells, VEGF is principally produced by podocytes in human kidneys and has been reported to be critical to the formation and integrity of the glomerular filtration barrier (GFB). There are two corresponding VEGF receptors (VEGFR) that belong to the tyrosine kinase family; although VEGFR-1 seems more significant to normal podocyte self-support in vivo, VEGFR-2 may play a dominant role in VEGF-signalling maintenance of glomerular and adjacent endothelial cells ${ }^{[49]}$. Canonical VEGF signalling within the renal glomerulus can be activated by VEGF secreted from podocytes, which then crosses the filtration barrier contrary to urinary flow to bind to VEGFR-2 expressed on the surface of glomerular endothelial cells. Connections between MPs and VEGF have been increasingly mentioned in recent years, which have increased the interest in these two interacting agents in $\mathrm{DN}$ pathogenesis. The role that the VEGF-A/VEGFR-2 system plays in DN is occasionally termed as a paradox: in incipient DN, renal expression of VEGF and VEGFR-2 has shown an increasing trend; however, at later phases, loss of podocytes reduced VEGF signalling, which led to vascular rarefication and renal fibrosis ${ }^{[50]}$. Whereas the overexpression of podocyte $\mathrm{VEGF}_{164}$ in adult transgenic mice can induce remarkably higher albuminuria positively correlated with renal VEGF, this overexpression also resulted in similar glomerular abnormalities reported in early $\mathrm{DN}[51]$. Intriguingly, VEGF- $\mathrm{A}_{165 \mathrm{~b}}$ is upregulated in $\mathrm{DN}$ patients and can restore glomerular endothelial glycocalyx to preserve renal function ${ }^{[52]}$. Such a paradox probably has something to do with both the expression of VEGF as several isoforms and the differences in renal phenotypes between humans and rodents ${ }^{[53]}$ Lymphocyte-derived microparticles (LMPs) have been discovered to exert antiangiogenic and proapoptotic effects by reducing the VEGF-A levels[54], which suggests that LMPs could be utilized in antiangiogenic therapeutic strategies for DN. Coincidentally, tumour-derived microparticles (TMPs) exert pro-angiogenic effects by inducing the mobilization and tumour homing of specific bone marrow derived cells (BMDCs), which contributes to the restart of tumour growth and can be blocked via substantially reducing the VEGF-A content of TMPs ${ }^{[55]}$.

\section{Therapeutic perspectives of MPs in DN}

Currently, conventional DN treatment includes ameliorating proteinuria and suboptimal maintenance of plasma glucose levels, with continuous renal replacement therapy (CRRT) and kidney transplantation in the end-stage renal disease 
(ESRD) phase. These measures are typically considered as the means of a last resort, which undoubtedly adds to the economic and social burdens for patients. These means can suspend but not prevent the development of DN. The list of MPs and their widely varied functions has dramatically extended in recent years, which highlights the therapeutic perspectives of MPs in clinical diagnosis and treatment of DN[56].

MPs have been utilized in pharmacologic delivery due to their submicron size along with the capacity to transfer materials among cells. As mentioned previously, MP composition can be determined by the cell of origin and the exogenous stimulus ${ }^{[5]}$ or, in a more pragmatic sense, modified with specific contents of therapeutic effects via artificial means ${ }^{[58]}$. These measures can preserve drug bio-stability and exert a synergistic influence on pathogenic factors, which might be more efficient than single drug administration. In addition, MPs can be designed to conform to drug-releasing patterns as required [59], which ensures cumulative and prolonged therapeutic effects without the need for repeated dose administration or the occurrence of adverse effects damaging liver or kidney function[60]. In contrast, concerning the different forms of MPs involved in the disease mechanisms, it is theoretically feasible to alleviate MP-driven deterioration in diseases by inhibiting relevant aspects of MP involvement, including their biogenesis, release, cell uptake and/or the transmission of specific contents to target cells. Quite a few inhibitors have been proposed in this regard, including glycoprotein IIb/IIIa antagonists[61], calpain inhibitors, ROCK inhibitors and calcium channel blockers[62]. Nevertheless, without widely practical applications to date, there are still impediments to be resolved. As to the protective effects of MPs mentioned above and the many other core cellular processes in which MPs may be active participants, interfering with these aspects might result in harmful off-target outcomes (See Figure 2).

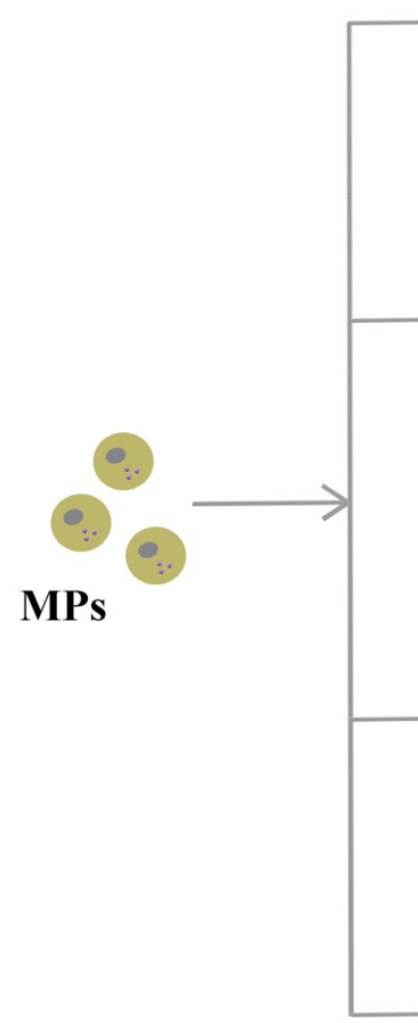

(1)

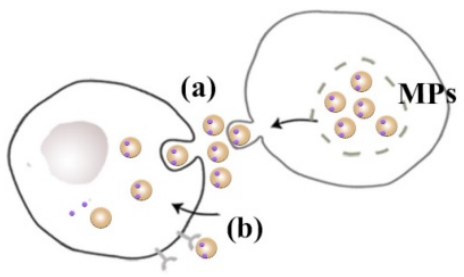

(2)
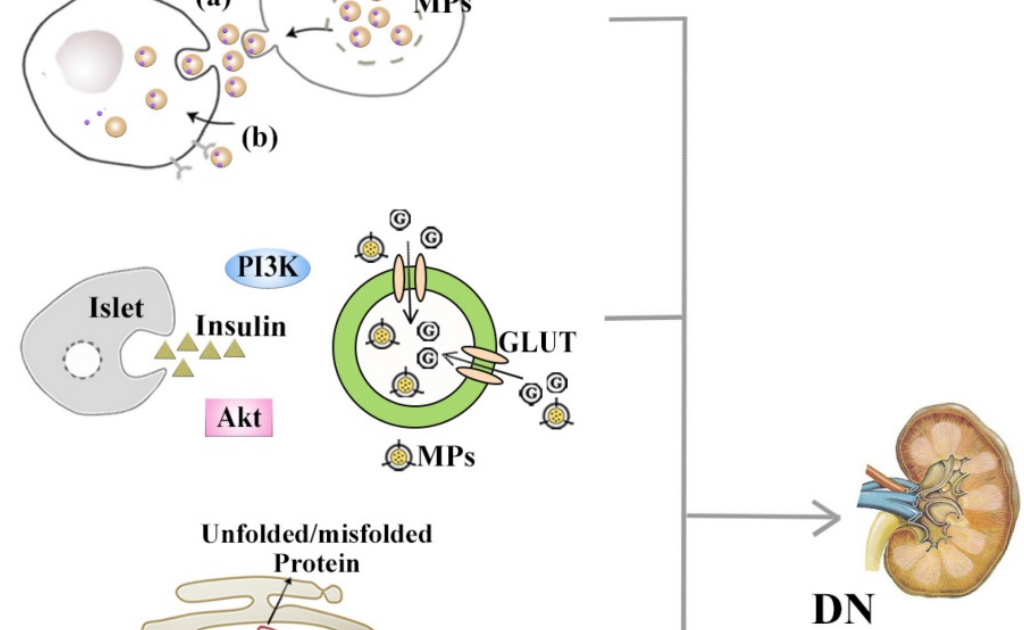

(3)

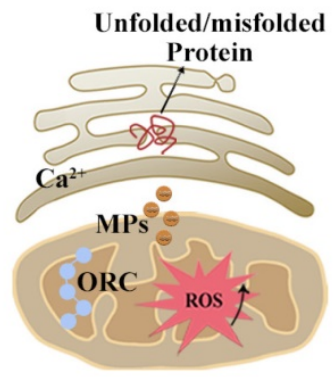

DN

(4)

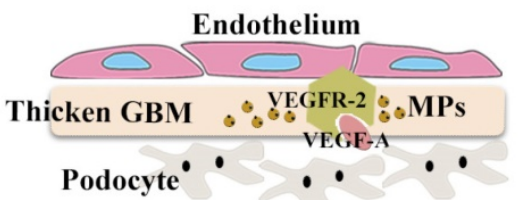

Figure 2. MPs are involved in DN pathogenesis. With demonstrable increases in DN-related pathological contexts, MPs might be involved in DN via the mechanisms, including (1) Mediating transcellular crosstalk mainly by (a) delivering their content to recipient cells and releasing these bioactive substances or (b) binding to the target cell receptors via surface molecules (namely, receptor-ligand interaction); (2) Promoting partial or systemic IR, possibly by influencing the insulin-related Akt/PI3K signalling and blocking glucose uptake by hindering glucose transporter (GLUT); (3) Increasing the production of cytosolic ROS, which activates organelle oxidative stress of mitochondria and ER and triggers inflammatory responses; (4) Exerting functions associated with angiogenesis and apoptosis and communicating between podocytes and glomerular endothelial cells via the VEGF-A/VEGFR-2 system. 


\section{Summary}

The medical literature abounds with studies claiming to focus specifically on either exosomes or MPs instead of a mixture of EVs, which has hindered extrapolation to this community and prompted the adoption of new guidelines including precise denomination methods and standard isolating protocols. As mentioned above, MPs are possibly produced and released during the onset and progression of DN and could, in turn, influence this whole process via several theorized mechanisms, which are so intertwined that usually one aspect is not considered to be completely separate from another. In spite of the breakthrough that has been achieved in this field, there is still a dearth of detailed knowledge regarding the underlying mechanisms. To fully delineate the biological roles and potential efficacy of MPs, further studies are required to establish standardized purification protocols and dissect the cellular mechanisms underlying the proposed aspects outlined above.

\section{Acknowledgements}

This work was supported by the National Natural Science Foundation of China (Grant 81470957), the Natural Science Foundation of Jiangsu Province (BK20141343), the Fundamental Research Funds for the Central Universities, the Jiangsu Province Six Talent Peaks Project (2015-WSN-002), the project for Jiangsu Provincial Medical Talent (ZDRCA2016077), the Jiangsu Province Ordinary University Graduate Research Innovation Project (KYZZ15-0061, SJZZ16-004), and the Clinical Medical Science Technology Special Project of Jiangsu Province (BL2014080).

\section{Competing Interests}

The authors have declared that no competing interest exists.

\section{References}

1. Wolf $\mathrm{P}$. The nature and significance of platelet products in human plasma. $\mathrm{BrJ}$ Haematol 1967; 13:269-288.

2. Samir ELA, Mager I, Breakefield XO, Wood MJ. Extracellular vesicles: biology and emerging therapeutic opportunities. Nat Rev Drug Discov 2013; 12:347-357.

3. Gould SJ, Raposo G. As we wait: coping with an imperfect nomenclature for extracellular vesicles. I Extracell Vesicles 2013; 2:20389.

4. Mause SF, Weber C. Microparticles: protagonists of a novel communication network for intercellular information exchange. Circ Res 2010; 107:1047-1057.

5. Burger D, Schock S, Thompson CS, Montezano AC, Hakim AM, Touyz RM. Microparticles: biomarkers and beyond. Clin Sci (Lond) 2013; 124:423-441.

6. Coleman ML, Sahai EA, Yeo M, Bosch M, Dewar A, Olson MF. Membrane blebbing during apoptosis results from caspase-mediated activation of ROCK I. Nat Cell Biol 2001; 3:339-345.

7. Desrochers LM, Bordeleau F, Reinhart-King CA, Cerione RA, Antonyak MA. Microvesicles provide a mechanism for intercellular communication by embryonic stem cells during embryo implantation. Nat Commun 2016; 7:11958.

8. Jansen F, Yang X, Hoyer FF, Paul K, Heiermann N, Becher MU, et al. Endothelial microparticle uptake in target cells is annexin I/phosphatidylserine receptor dependent and prevents apoptosis. Arterioscler Thromb Vasc Biol 2012; 32:1925-1935.
9. Niessen A, Heyder P, Krienke S, Blank N, Tykocinski LO, Lorenz HM, et al. Apoptotic-cell-derived membrane microparticles and IFN-alpha induce an inflammatory immune response. J Cell Sci 2015; 128:2443-2453.

10. Han CZ, Juncadella IJ, Kinchen JM, Buckley MW, Klibanov AL, Dryden K, et al. Macrophages redirect phagocytosis by non-professional phagocytes and influence inflammation. Nature 2016; 539:570-574.

11. Rank A, Nieuwland R, Crispin A, Grutzner S, Iberer M, Toth B, et al. Clearance of platelet microparticles in vivo. Platelets 2011; 22:111-116.

12. Augustine D, Ayers LV, Lima E, Newton L, Lewandowski AJ, Davis EF, et al. Dynamic release and clearance of circulating microparticles during cardiac stress. Circ Res 2014; 114:109-113.

13. Shantsila E, Montoro-Garcia S, Gallego P, Lip GY. Circulating microparticles: challenges and perspectives of flow cytometric assessment. Thromb Haemost 2014; 111:1009-1014.

14. Leslie RD, Kolb H, Schloot NC, Buzzetti R, Mauricio D, De Leiva A, et al. Diabetes classification: grey zones, sound and smoke: Action LADA 1. Diabetes Metab Res Rev. 2008; 24(7):511-9.

15. Mayer-Davis EJ, Lawrence JM, Dabelea D, Divers J, Isom S, Dolan L, et al. Incidence Trends of Type 1 and Type 2 Diabetes among Youths, 2002-2012. N Engl J Med 2017; 376:1419-1429.

16. Chiva-Blanch G, Suades R, Padro T, Vilahur G, Pena E, Ybarra I, et al. Microparticle Shedding by Erythrocytes, Monocytes and Vascular Smooth Muscular Cells Is Reduced by Aspirin in Diabetic Patients. Rev Esp Cardiol (Engl Ed) 2016; 69:672-680.

17. Feng B, Chen Y, Luo Y, Chen M, Li X, Ni Y. Circulating level of microparticles and their correlation with arterial elasticity and endothelium-dependent dilation in patients with type 2 diabetes mellitus. Atherosclerosis 2010; 208:264-269.

18. Alexandru N, Badila E, Weiss E, Cochior D, Stepien E, Georgescu A. Vascular complications in diabetes: Microparticles and microparticle associated microRNAs as active players. Biochem Biophys Res Commun 2016; 472:1-10.

19. Zhang L, Long J, Jiang W, Shi $Y$, He X, Zhou Z, et al. Trends in Chronic Kidney Disease in China. N Engl J Med 2016; 375:905-906.

20. Perkins BA, Ficociello LH, Roshan B, Warram JH, Krolewski AS. In patients with type 1 diabetes and new-onset microalbuminuria the development of advanced chronic kidney disease may not require progression to proteinuria. Kidney Int 2010; 77:57-64.

21. Li S, Wei J, Zhang C, Li X, Meng W, Mo X, et al. Cell-Derived Microparticles in Patients with Type 2 Diabetes Mellitus: a Systematic Review and Meta-Analysis. Cell Physiol Biochem 2016; 39:2439-2450.

22. Lu GY, Xu RJ, Zhang SH, Qiao O, Shen L, Li M, et al. Alteration of circulatory platelet microparticles and endothelial microparticles in patients with chronic kidney disease. Int J Clin Exp Med 2015; 8:16704-16708.

23. Lytvyn Y, Xiao F, Kennedy CR, Perkins BA, Reich HN, Scholey JW, et al. Assessment of urinary microparticles in normotensive patients with type 1 diabetes. Diabetologia 2017; 60:581-584

24. Burger D, Thibodeau JF, Holterman CE, Burns KD, Touyz RM, Kennedy CR. Urinary podocyte microparticles identify prealbuminuric diabetic glomerular injury. I Am Soc Nephrol 2014; 25:1401-1407.

25. Kwon SH, Woollard JR, Saad A, Garovic VD, Zand L, Jordan KL, et al. Elevated urinary podocyte-derived extracellular microvesicles in renovascular hypertensive patients. Nephrol Dial Transplant 2016; 0:1-7.

26. Sun AL, Deng JT, Guan GJ, Chen SH, Liu YT, Cheng J, et al. Dipeptidyl peptidase-IV is a potential molecular biomarker in diabetic kidney disease. Diab Vasc Dis Res 2012; 9:301-308.

27. Ouyang C, You J, Xie Z. The interplay between autophagy and apoptosis in the diabetic heart. J Mol Cell Cardiol 2014; 71:71-80.

28. Perkovic V, Heerspink HL, Chalmers J, Woodward M, Jun M, Li Q, et al. Intensive glucose control improves kidney outcomes in patients with type 2 diabetes. Kidney Int 2013; 83:517-523.

29. Eirin A, Zhu XY, Puranik AS, Tang H, McGurren KA, van Wijnen AJ, et al. Mesenchymal stem cell-derived extracellular vesicles attenuate kidney inflammation. Kidney Int 2017; 92(1):114-124.

30. Eguchi A, Mulya A, Lazic M, Radhakrishnan D, Berk MP, Povero D, et al. Microparticles release by adipocytes act as "find-me" signals to promote macrophage migration. PLoS One 2015; 10(4):e0123110.

31. Eyre J, Burton JO, Saleem MA, Mathieson PW, Topham PS, Brunskill NJ. Monocyte- and endothelial-derived microparticles induce an inflammatory phenotype in human podocytes. Nephron Exp Nephrol 2011; 119:e58-66.

32. Jansen F, Yang X, Hoelscher M, Cattelan A, Schmitz T, Proebsting S, et al. Endothelial microparticle-mediated transfer of MicroRNA-126 promotes vascular endothelial cell repair via SPRED1 and is abrogated in glucose-damaged endothelial microparticles. Circulation 2013; 128:2026-2038.

33. Lin CL, Lee PH, Hsu YC, Lei CC, Ko JY, Chuang PC, et al. MicroRNA-29a promotion of nephrin acetylation ameliorates hyperglycemia-induced podocyte dysfunction. I Am Soc Nephrol 2014; 25:1698-1709.

34. Figliolini F, Cantaluppi V, De Lena M, Beltramo S, Romagnoli R, Salizzoni M, et al. Isolation, characterization and potential role in beta cell-endothelium cross-talk of extracellular vesicles released from human pancreatic islets. PLoS One 2014; 9:e102521.

35. Burger D, Turner M, Munkonda MN, Touyz RM. Endothelial Microparticle-Derived Reactive Oxygen Species: Role in Endothelial Signaling and Vascular Function. Oxid Med Cell Longev 2016; 2016:5047954. 
36. Karalliedde J, Gnudi L. Diabetes mellitus, a complex and heterogeneous disease, and the role of insulin resistance as a determinant of diabetic kidney disease. Nephrol Dial Transplant 2016; 31:206-213.

37. Artunc F, Schleicher E, Weigert C, Fritsche A, Stefan N, Haring HU. The impact of insulin resistance on the kidney and vasculature. Nat Rev Nephrol 2016; 12:721-737.

38. Kranendonk ME, Visseren FL, van Herwaarden JA, Nolte-'t Hoen EN, de Jager $\mathrm{W}$, Wauben $\mathrm{MH}$, et al. Effect of extracellular vesicles of human adipose tissue on insulin signaling in liver and muscle cells. Obesity (Silver Spring) 2014; 22:2216-2223.

39. Choi Y, Kwon Y, Kim DK, Jeon J, Jang SC, Wang T, et al. Gut microbe-derived extracellular vesicles induce insulin resistance, thereby impairing glucose metabolism in skeletal muscle. Sci Rep 2015; 5:15878.

40. Jalabert A, Vial G, Guay C, Wiklander OP, Nordin JZ, Aswad H, et al. Exosome-like vesicles released from lipid-induced insulin-resistant muscles modulate gene expression and proliferation of beta recipient cells in mice. Diabetologia 2016; 59:1049-1058.

41. Kranendonk ME, Visseren FL, van Balkom BW, Nolte-'t Hoen EN, van Herwaarden JA, de Jager W, et al. Human adipocyte extracellular vesicles in reciprocal signaling between adipocytes and macrophages. Obesity (Silver Spring) 2014; 22:1296-1308.

42. Zhang Y, Shi L, Mei H, Zhang J, Zhu Y, Han X, et al. Inflamed macrophage microvesicles induce insulin resistance in human adipocytes. Nutr Metab (Lond) $2015 ; 12: 21$

43. Jung C, Shemyakin A, Bohm F, Pernow J. Endothelial microparticles in patients with insulin resistance. Diabetes Metab 2009; 35:71-73.

44. Qi H, Casalena G, Shi S, Yu L, Ebefors K, Sun Y, et al. Glomerular Endothelial Mitochondrial Dysfunction Is Essential and Characteristic of Diabetic Kidney Disease Susceptibility. Diabetes 2017; 66:763-778.

45. Lindenmeyer MT, Rastaldi MP, Ikehata M, Neusser MA, Kretzler M, Cohen $\mathrm{CD}$, et al. Proteinuria and hyperglycemia induce endoplasmic reticulum stress. J Am Soc Nephrol 2008; 19:2225-2236.

46. Inzaugarat ME, Wree A, Feldstein AE. Hepatocyte mitochondrial DNA released in microparticles and toll-like receptor 9 activation: A link between lipotoxicity and inflammation during nonalcoholic steatohepatitis. Hepatology 2016; 64:669-671.

47. Safiedeen Z, Rodriguez-Gomez I, Vergori L, Soleti R, Vaithilingam D, Douma I et al. Temporal Cross talk Between Endoplasmic Reticulum and Mitochondria Regulates Oxidative Stress and Mediates Microparticle-Induced Endothelial Dysfunction. Antioxid Redox Signal 2016; 26:15-27.

48. Hagberg CE, Mehlem A, Falkevall A, Muhl L, Fam BC, Ortsater H, et al. Targeting VEGF-B as a novel treatment for insulin resistance and type 2 diabetes. Nature 2012; 490:426-430.

49. Sison K, Eremina V, Baelde H, Min W, Hirashima M, Fantus IG, et al. Glomerular structure and function require paracrine, not autocrine, VEGF-VEGFR-2 signaling. J Am Soc Nephrol 2010; 21:1691-1701.

50. Fu J, Lee K, Chuang PY, Liu Z, He JC. Glomerular endothelial cell injury and cross talk in diabetic kidney disease. Am J Physiol Renal Physiol 2015; 308:F287-297.

51. Veron D, Reidy KJ, Bertuccio C, Teichman J, Villegas G, Jimenez J, et al. Overexpression of VEGF-A in podocytes of adult mice causes glomerular disease. Kidney Int 2010; 77:989-999.

52. Oltean S, Qiu Y, Ferguson JK, Stevens M, Neal C, Russell A, et al. Vascular Endothelial Growth Factor-A165b Is Protective and Restores Endothelial Glycocalyx in Diabetic Nephropathy. J Am Soc Nephrol 2015; 26:1889-1904.

53. Majumder S, Advani A. VEGF and the diabetic kidney: More than too much of a good thing. J Diabetes Complications 2017; 31(1):273-279.

54. Yang C, Gagnon C, Hou X, Hardy P. Low density lipoprotein receptor mediates anti-VEGF effect of lymphocyte T-derived microparticles in Lewis lung carcinoma cells. Cancer Biol Ther 2010;:448-456.

55. Munster M, Fremder E, Miller V, Ben-Tsedek N, Davidi S, Scherer SJ, et al. Anti-VEGF-A affects the angiogenic properties of tumor-derived microparticles. PLoS One 2014; 9:e95983.

56. Bale S, Khurana A, Reddy AS, Singh M, Godugu C. Overview on Therapeutic Applications of Microparticulate Drug Delivery Systems. Crit Rev Ther Drug Carrier Syst 2016; 33:309-361.

57. Tang $\mathrm{K}$, Zhang $\mathrm{Y}$, Zhang $\mathrm{H}$, Xu P, Liu J, Ma J, et al. Delivery of chemotherapeutic drugs in tumour cell-derived microparticles. Nat Commun 2012; 3:1282.

58. Mizrak A, Bolukbasi MF, Ozdener GB, Brenner GJ, Madlener S, Erkan EP, et al. Genetically engineered microvesicles carrying suicide mRNA/protein inhibit schwannoma tumor growth. Mol Ther 2013; 21:101-108.

59. Madhusudhan S, Panda AK, Parimalakrishnan S, Manavalan R, Manna PK. Design, in vitro and in vivo evaluation of glipizide Eudragit microparticles. $J$ Microencapsul 2010; 27:281-291.

60. Abadi SS, Moin A, Veerabhadrappa GH. Review Article: Fabricated Microparticles: An Innovative Method to Minimize the Side Effects of NSAIDs in Arthritis. Crit Rev Ther Drug Carrier Syst 2016; 33:433-488.

61. $\mathrm{Li} \mathrm{X}$, Cong $\mathrm{H}$. Platelet-derived microparticles and the potential of glycoprotein $\mathrm{IIb} / \mathrm{III}$ antagonists in treating acute coronary syndrome. Tex Heart Inst J 2009; 36:134-139.

62. Roseblade A, Luk F, Ung A, Bebawy M. Targeting microparticle biogenesis: a novel approach to the circumvention of cancer multidrug resistance. Curr Cancer Drug Targets 2015; 15:205-214. 